\title{
High blood pressure and lost EDHF response in redox dead Cys42Ser PKGla knock-in mouse
}

\author{
Oleksandra Prysyazhna, Olena Rudyk, Philip Eaton* \\ From 5th International Conference on cGMP: Generators, Effectors and Therapeutic Implications \\ Halle, Germany. 24-26 June 2011
}

\begin{abstract}
Background
We previously showed PKGI $\alpha$ forms an interprotein disulfide between its two subunits in response to oxidants such as hydrogen peroxide $\left(\mathrm{H}_{2} \mathrm{O}_{2}\right)$. This activates PKGIa independently of the classical NO-cGMP pathway. The oxidative activation of PKGI $\alpha$ may contribute to the endothelium-derived hyperpolarising factor (EDHF) phenomenon, especially as oxidant species such as $\mathrm{H}_{2} \mathrm{O}_{2}$ have been implicated as this factor.
\end{abstract}

\section{Results}

To investigate this further we generated a Cys42Ser PKGI $\alpha$ knock-in (KI) mouse line. Immunoblotting confirmed that tissues from KI mice express PKGI $\alpha$ at the same level as wild type (WT). However, when KI hearts were perfused in Langendorff mode and exposed to $\mathrm{H}_{2} \mathrm{O}_{2}(50 \mu \mathrm{M}, 10 \mathrm{~min})$ they did not form a disulfide dimer as anticipated, whereas WT hearts did. Similarly aorta and mesenteric vessels from WT, but not KI, mice formed disulfide in response to $\mathrm{H}_{2} \mathrm{O}_{2}$. To examine the functional contribution of PKGI $\alpha$ disulfide dimerisation to oxidant-induced vasodilation, we compared the response of isolated rings of thoracic aorta and second order mesenteric arteries from WT or KI mice to $\mathrm{H}_{2} \mathrm{O}_{2}$. WT or KI rings of aorta that were preconstricted with EC80 phenylephrine $(1 \mu \mathrm{M})$ and then serially exposed to increasing concentration of $\mathrm{H}_{2} \mathrm{O}_{2}(\mathrm{n}=3,10$ rings per group). $\mathrm{KI}$ aortas were resistant to $\mathrm{H}_{2} \mathrm{O}_{2}$-induced relaxation, showing a $\sim 40 \%$ deficit in their maximal response. Second-order mesenteric arteries were preconstricted with EC80 u46619 $(0.5 \mu \mathrm{M})$ and then relaxed by exposure to increasing concentrations of $\mathrm{H}_{2} \mathrm{O}_{2}$, observing a significant rightward shift (insensitivity) in the KI doseresponse compared to WT. To assess whether PKGIa disulfide activation contributes to the EDHF phenomenon we compared WT and KI relaxations to acetylcholine chloride $(\mathrm{ACh}, 1 \mu \mathrm{M})$ in vessels with or without inhibition of NO (L-NAME $300 \mu \mathrm{M}, 30 \mathrm{~min}$ ) and prostanoid (indomethacin $10 \mu \mathrm{M}, 30 \mathrm{~min}$ ) synthesis. The EDHF response was absent in aorta regardless of genotype. In contrast the EDHF response accounted for 30\% of total ACh relaxation in WT mesenterics. KI mesenteric EDHF relaxation was absent and total ACh response was significantly attenuated. To assess the importance of these events in vivo we used blood pressure telemetry monitoring. Blood pressure (SAP, MAP and DAP) was significantly higher in KI mice than littermate WTs.

\section{Conclusion}

PKGI $\alpha$ disulfide formation is a significant component of oxidant-induced vasodilation, consistent with this being a major component of the EDHF phenomenon. Furthermore, this mechanism operates basally to control blood pressure, as its genetic removal in the KI results in hypertension.

Published: 1 August 2011

doi:10.1186/1471-2210-11-S1-O22

Cite this article as: Prysyazhna et al:: High blood pressure and lost EDHF response in redox dead Cys42Ser PKGla knock-in mouse. BMC Pharmacology 2011 11(Suppl 1):O22. 